\section{Hermanns, Wim: GOT - Ganzheitliche Osteopathische Therapie}

Hippokrates: Stuttgart; 2007. 144 Seiten, 153 Abbildungen, ISBN 978-3-8304-5352-9, $€ 49,95$

$\mathrm{H}$ ermanns bietet in seiner nur 144 Seiten umfassenden Broschur eine sehr persönliche, weniger wissenschaftliche als empathische Beschreibung der GOT. Der Begriff „ganzheitliche osteopathische Therapie“" ist seine freie Übersetzung der ursprünglichen Bezeichnung „general osteopathic treatment". Die GOT, besser bekannt unter der deutschen Abkürzung $\mathrm{AOB}$ (allgemeine osteopathische Behandlung), wurde von Littlejohn begründet und von Wernham weiterentwickelt. Sie stellt eine Methode zur Untersuchung, Diagnostik und Behandlung des gesamten Bewegungsapparates dar. Mit kreisenden Bewegungen werden (nahezu) alle Gelenke in einem auf den Patienten angepassten Rhythmus bewegt. Dabei wird die GOT immer nach dem gleichen Behandlungsregime durchgeführt.

Beseelt von den Ideen Littlejohns und Wernhams bricht Hermanns eine Lanze für eine stärkere Akzeptanz und Würdigung der GOT. Besonders zu Beginn ist das Werk durchsetzt von philosophischen Gedanken zu seiner Auffassung von Medizin im Allgemeinen und zur Osteopathie im Speziellen. Leider wird dabei sehr unscharf mit dem Wort „ganzheitlich“ (als freie Übersetzung des Begriffs „general“) umgegangen. Ein unklarer und schwammiger Begriff, jeder Therapeut möchte ganzheitlich arbeiten, aber jeder versteht etwas anders darunter.

\section{Aufbau}

Die ersten sieben Kapitel befassen sich mit den Grundlagen der GOT. Hermanns schlägt den Bogen von philosophischen Gedanken zur Geschichte der Medizin und den Prinzipien der Osteopathie im Allgemeinen über kurze Abrisse der Biographien Stills, Littlejohns und Wernhams zu den Prinzipien der GOT.

Im folgenden Kapitel stellt der Autor die Common Compensatory Pattern nach Zink, das modifizierte Common Pelvic Pattern nach Wernham sowie die posturalen Modelle nach Hall als mögliche Untersuchungsmethoden vor.

Daran schließen sich die Kapitel über die Behandlungssequenzen an. Es werden nacheinander Techniken in Rückenlage, in Bauchlage, dann in Seitenlage und sitzender Position beschrieben. Leider sind viele der gut in den Text eingearbeiteten schwarz-weißen Fotos unscharf.
Abschließend beschreibt Hermanns mögliche Kombinationen der GOT mit anderen Techniken, insbes. der MET und den Triggerpunktbehandlungen.

\section{Fazit}

Trotz oder vielleicht gerade wegen der subjektiven Betrachtungsweise Hermanns ist die vorliegende Broschur lesenswert. Sie bietet in Kürze einen interessanten Einblick in die Denk- und Behandlungsweise innerhalb der GOT.

Dirk Luthin, Hameln 